\title{
A systematic understanding of orbital energy shift in polar solvent
}

\section{$\operatorname{AUTHOR}(S)$ :}

lida, Kenji; Yokogawa, Daisuke; Sato, Hirofumi; Sakaki, Shigeyoshi

\section{CITATION:}

lida, Kenji ... [et al]. A systematic understanding of orbital energy shift in polar solvent. The Journal of chemical physics 2009, 130(4): 044107.

\section{ISSUE DATE:}

2009-01-28

URL:

http://hdl.handle.net/2433/123548

\section{RIGHT:}

Copyright 2009 American Institute of Physics. This article may be downloaded for personal use only. Any other use requires prior permission of the author and the American Institute of Physics. The following article appeared in The Journal of Chemical Physics 130, 044107 (2009) and may be found at http://link.aip.org/link/JCPSA6/v130/i4/p044107/s1 


\title{
A systematic understanding of orbital energy shift in polar solvent
}

\author{
Kenji lida, Daisuke Yokogawa, Hirofumi Sato, a) and Shigeyoshi Sakaki \\ Department of Molecular Engineering, Kyoto University, Kyoto 615-8510, Japan
}

(Received 17 September 2008; accepted 18 December 2008; published online 29 January 2009)

\begin{abstract}
The orbital energy of molecule is significantly shifted upon going from gas phase to solution phase. Based on Koopmans' theorem, the shift should be related to the change of ionization potential. However, the computed shift looks usually random and clear understanding has not been attained yet. Furthermore it is obtained only after solving complicated equations. In this study, we report a systematic framework for understanding the orbital energy shift by solvation effect and simple approximate formulae are presented. (C) 2009 American Institute of Physics.
\end{abstract}

[DOI: $10.1063 / 1.3068531]$

\section{INTRODUCTION}

Orbital energy $(\varepsilon)$ is one of the most important quantities to characterize electronic structure of molecule. For example, $\varepsilon$ plays a crucial role to describe the reactivity in the frontier orbital theory. $\varepsilon$ is also deeply related to the molecular property such as ionization potential (IP) through Koopmans' theorem, ${ }^{1}$ although $\varepsilon$ is not physically measurable. Recently, direct observation of ionization and excitation energy becomes experimentally available for solvated molecule, ${ }^{2,3}$ and the observed values often exhibit significant change from gas-phase ones. However systematic understanding has not been established yet.

In fact, it is also well known that computed $\varepsilon$ in solution phase is different from that in gas phase due to the interaction between solute and solvent. ${ }^{4-7}$ The electronic structure of solvated molecule can be calculated using solvation theory such as the family of reference interaction site modelself-consistent field ${ }^{8-10}$ (RISM-SCF) and polarizable continuum model (PCM). ${ }^{11}$ However, the obtained shifts look often random; $\varepsilon$ sometimes increases and sometimes decreases. Figure 1 illustrates the shifts of orbital energies of acetamide in aqueous solution computed by PCM. It looks lacking in orderliness, namely, classifications such as corevalence or $\sigma-\pi$ orbital seem nothing short of impossible. It is also noted that the shifts are obtained only after solving complicated modified Schrödinger equation. Unfortunately, we could not find any literatures for systematic study of the solvation shift although several comprehensive reports on polarization of solvated molecule have been presented. ${ }^{12,13}$ In other words, we can explain the mechanism of the shift neither logically nor comprehensively, and the understanding of the solvation shift of $\varepsilon$ has not been systematized at all.

In this study we propose a systematic framework for understanding the orbital energy shift due to solvation effect. Since the electronic polarization of a molecule is coupled with solvent reaction field, the shift could be understood

\footnotetext{
${ }^{a)}$ Author to whom correspondence should be addressed. FAX: +81-75-3832799. Electronic mail: hirofumi@moleng.kyoto-u.ac.jp.
}

from the view point of dielectric continuum theory. With the aid of the theory, the shift is systematized and several simple formulae are presented to rationalize it.

\section{COMPUTATIONAL DETAILS}

All the calculations were carried out at Hartree-Fock (HF) level using 6-311++ $\mathrm{G}^{* *}$ basis set. ${ }^{14}$ The computations were carried out with the gas-phase optimized geometries because we would like to exclude the geometrical contribution to the orbital energy shift. We examined the following 19 organic compounds; acetamide, acetic acid, acetone, ammonia, dimethyl ether, dimethyl sulfide, formaldehyde, formamide, hydrogen sulfide, methanethiol, methanol, methyl amine, $N$-methyl formamide, phosphoric acid, propene, pyridine, pyrole, sulfuric acid, and water. They were taken from Ref. 15 and supply 265 molecular orbitals (MOs) including core orbitals (all of them were considered in this study). Several atomic ions $\left(\mathrm{Li}^{+}, \mathrm{Na}^{+}, \mathrm{K}^{+}, \mathrm{F}^{-}, \mathrm{Cl}^{-}, \mathrm{Br}^{-}, \mathrm{I}^{-}\right)$ were also studied.

Solvation effect was taken into account by RISM-SCFSEDD (spatial electron density distribution) (Ref. 10) and PCM. ${ }^{11}$ The RISM-SCF-SEDD is a hybrid method of MO theory and statistical mechanics, an integral equation theory for molecular liquid. Similar to QM/MM, the method determines the electronic structure of a solute as well as the solvent distribution around it in a self-consistent manner, and successfully applied to various chemical processes in solution phase. The method provides microscopic information of the solvation structure in terms of the pair correlation function (PCF). A typical example is illustrated in Fig. 2. The first peak between $\mathrm{O}$ (acetamide)-H(solvent water) found in $2 \AA$ represents the hydrogen bonding. The strong electrostatic field on the oxygen atom in acetamide generated by this localized interaction can be dealt with by RISM-SCF-SEDD method.

The RISM equation was solved with hypernetted chain closure $^{16}$ at $T=298.15 \mathrm{~K}$. On solving the portion of RISM procedure, the Lennard-Jones parameters for the solute molecules were taken from Refs. 17-21. SPC-like model of water was employed ${ }^{22}$ with a correction of the LennardJones parameters of the hydrogen sites $(\sigma=1.0 \AA$, 


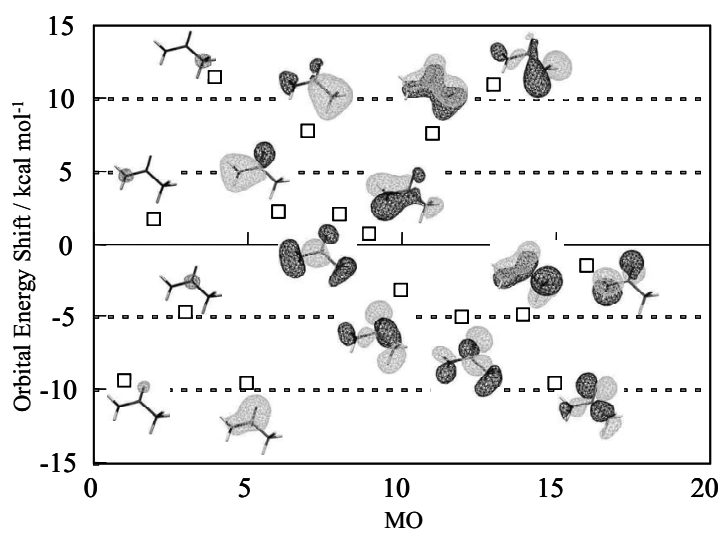

FIG. 1. Shift of MO energy by solvation effect computed by PCM. HF with 6-311++ $\mathrm{G}^{* *}$ was employed.

$\epsilon=0.056 \mathrm{kcal} \mathrm{mol}^{-1}$ ) under the condition of $1.0 \mathrm{~g} / \mathrm{cm}^{3}$. Acetonitrile $^{23}$ and methanol ${ }^{24,25}$ were also employed as solvent with 0.786 and $0.792 \mathrm{~g} / \mathrm{cm}^{3}$ of density, respectively. All the parameters are summarized in Table I. PCM computations were performed with the standard parameters implemented in GAMESS program package ${ }^{26}$ while the parameters for atomic species (ions) and acetonitrile solvent were taken from GAUSSIAN $03 .^{27}$ RISM-SCF-SEDD calculations also carried out with GAMESS modified by us. It should be emphasized that a general framework of the understanding is independent on the choice of the solvation models as will be shown below, although two different methods, RISM-SCFSEDD and PCM, were employed to compute the electronic structures of solvated molecules in this study.

\section{THEORETICAL CONSIDERATION OF ORBITAL SHIFT}

\section{A. General theory and electrostatic interaction}

The orbital energy in gas phase $\left(\varepsilon_{i}^{0}\right)$ related to $\mathrm{MO} \psi_{i}^{0}$ is given by standard Fock operator $\hat{F}^{0}$ as follows:

$$
\varepsilon_{i}^{0}=\left\langle\psi_{i}^{0}\left|\hat{F}^{0}\right| \psi_{i}^{0}\right\rangle .
$$

In solution phase, electrostatic interaction is formed between solute and solvent and the potential field from solvent, $\hat{V}$ is added to the operator,

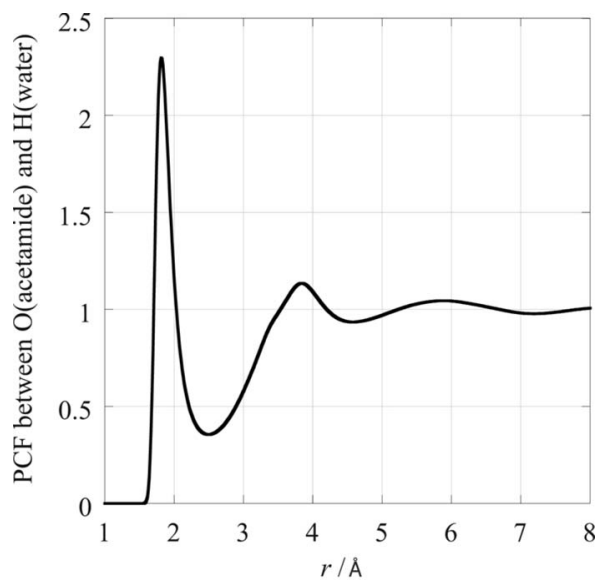

FIG. 2. PCF between oxygen (acetamide) and hydrogen (solvent water) obtained by RISM-SCF-SEDD computations.
TABLE I. Lennard-Jones parameters.

\begin{tabular}{|c|c|c|}
\hline & $\sigma / \AA$ & $\epsilon / \mathrm{kcal} \mathrm{mol}^{-1}$ \\
\hline \multicolumn{3}{|c|}{ Solute } \\
\hline C & 3.296 & 0.1200 \\
\hline $\mathrm{O}$ & 3.166 & 0.1550 \\
\hline $\mathrm{N}$ & 3.250 & 0.1700 \\
\hline S & 4.000 & 0.2000 \\
\hline $\mathrm{H}$ & 1.000 & 0.0560 \\
\hline $\mathrm{Li}$ & 1.394 & 0.3707 \\
\hline $\mathrm{Na}$ & 2.274 & 0.3206 \\
\hline K & 3.154 & 0.3060 \\
\hline $\mathrm{F}$ & 2.720 & 0.3480 \\
\hline $\mathrm{Cl}$ & 3.620 & 0.4480 \\
\hline $\mathrm{Br}$ & 3.900 & 0.5290 \\
\hline I & 4.320 & 0.6330 \\
\hline \multicolumn{3}{|c|}{$\mathrm{H}_{2} \mathrm{O}$ solvent } \\
\hline $\mathrm{O}$ & 3.166 & 0.1550 \\
\hline \multirow[t]{2}{*}{$\mathrm{H}$} & 1.000 & 0.0560 \\
\hline & $\mathrm{CH}_{3} \mathrm{OH}$ & \\
\hline $\mathrm{Me}$ & 3.775 & 0.2070 \\
\hline $\mathrm{O}$ & 3.070 & 0.1700 \\
\hline \multirow[t]{2}{*}{$\mathrm{H}$} & 1.000 & 0.0460 \\
\hline & $\mathrm{CH}_{3} \mathrm{Cr}$ & \\
\hline $\mathrm{Me}$ & 3.775 & 0.2070 \\
\hline C & 3.650 & 0.1500 \\
\hline $\mathrm{N}$ & 3.200 & 0.1700 \\
\hline
\end{tabular}

$$
\varepsilon_{i}=\left\langle\psi_{i}|\hat{F}+\hat{V}| \psi_{i}\right\rangle
$$

where $\psi_{i}$ and $\varepsilon_{i}$ are the MO and its energy of solvated molecule, respectively. It is noted that both of $\hat{F}$ and $\hat{V}$ are the functional of MOs, which change upon transferring from gas phase to solution phase,

$$
\begin{aligned}
& \hat{F}=\hat{F}^{0}+\Delta \hat{F}, \\
& \hat{V}=\hat{V}^{0}+\Delta \hat{V},
\end{aligned}
$$

where both of $\hat{F}^{0}$ and $\hat{V}^{0}$ are associated with the electronic wave function of the solute molecule in gas phase. Thus, the orbital energy shift by solvation $\left(\Delta \varepsilon_{i}\right)$ is given by

$$
\Delta \varepsilon_{i}=\varepsilon_{i}-\varepsilon_{i}^{0}=\varepsilon_{i}^{\mathrm{UV}}+\varepsilon_{i}^{\mathrm{rlx}}+\varepsilon_{i}^{\mathrm{ee}}+\varepsilon_{i}^{\mathrm{wf}},
$$

where

$$
\begin{gathered}
\varepsilon_{i}^{\mathrm{UV}} \equiv\left\langle\psi_{i}^{0}\left|\hat{V}^{0}\right| \psi_{i}^{0}\right\rangle, \\
\varepsilon_{i}^{\mathrm{rlx}} \equiv\left\langle\psi_{i}^{0}|\Delta \hat{V}| \psi_{i}^{0}\right\rangle, \\
\varepsilon_{i}^{\mathrm{ee}} \equiv\left\langle\psi_{i}^{0}|\Delta \hat{F}| \psi_{i}^{0}\right\rangle .
\end{gathered}
$$

$\varepsilon_{i}^{\mathrm{wf}}$ is all the other contributions arising from the change in wave function, $\psi_{i}-\psi_{i}^{0} \equiv \Delta \psi_{i}$. This term can be reduced by using orthogonality of MOs, ${ }^{28}$ 


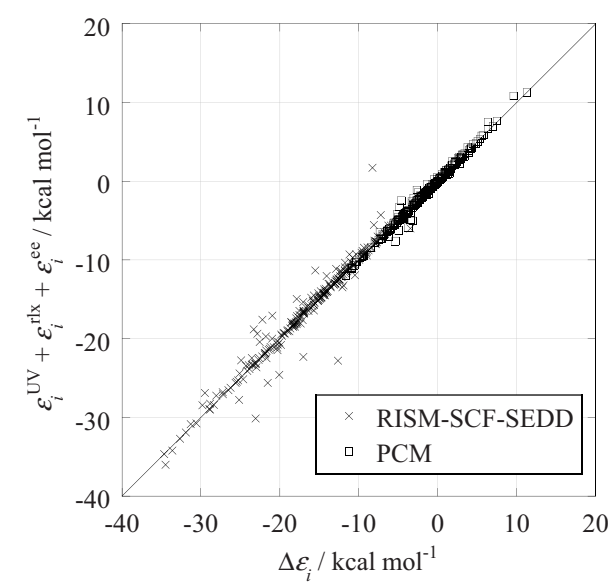

FIG. 3. Comparison between the exact values $\left(\Delta \varepsilon_{i}\right)$ and Eq. (8).

$$
\begin{aligned}
\varepsilon_{i}^{\mathrm{wf}}= & \left\langle\psi_{i}|\hat{F}+\hat{V}| \psi_{i}\right\rangle-\left\langle\psi_{i}^{0}|\hat{F}+\hat{V}| \psi_{i}^{0}\right\rangle=2\left\langle\Delta \psi_{i}|\hat{F}+\hat{V}| \psi_{i}\right\rangle \\
& -\left\langle\Delta \psi_{i}|\hat{F}+\hat{V}| \Delta \psi_{i}\right\rangle=2 \varepsilon_{i}\left\langle\Delta \psi_{i} \mid \psi_{i}\right\rangle+\mathcal{O}\left\{\left(\Delta \psi_{i}\right)^{2}\right\} \\
= & 2 \varepsilon_{i}\left\langle\Delta \psi_{i} \mid \psi_{i}^{0}\right\rangle+\mathcal{O}\left\{\left(\Delta \psi_{i}\right)^{2}\right\} \simeq 0 .
\end{aligned}
$$

$\Delta \varepsilon_{i}$ is then expected to be approximated as follows:

$$
\Delta \varepsilon_{i} \simeq \varepsilon_{i}^{\mathrm{UV}}+\varepsilon_{i}^{\mathrm{rlx}}+\varepsilon_{i}^{\mathrm{ee}} .
$$

The quantities of both sides of this equation were separately obtained for comparison: the left-hand side is the difference computed by subtracting gas phase $\varepsilon_{i}^{0}$ from $\varepsilon_{i}$ directly obtained with PCM or RISM-SCF-SEDD methods. The righthand side is the sum of matrix components that are related to PCM or RISM-SCF-SEDD procedure. As shown in Fig. 3, the contribution from $\varepsilon_{i}^{\mathrm{wf}}$ is surely negligible and the orbital energy shift, $\Delta \varepsilon_{i}$, is well approximated by the sum of the three terms, namely, right-hand side of Eq. (8) in almost all cases.

Let us proceed to further simplification of the relation. By expanding about arbitrary point, $\varepsilon_{i}^{\mathrm{UV}}$ is rewritten as follows:

$$
\varepsilon_{i}^{\mathrm{UV}}=\left\langle\psi_{i}^{0}\left|\hat{V}^{0}\right| \psi_{i}^{0}\right\rangle=Q_{i} V^{0}+\boldsymbol{\mu}_{i}^{0} \cdot \mathbf{R}^{0}+\cdots,
$$

where $Q_{i}$ and $\boldsymbol{\mu}_{i}^{0}$ are the total charge and dipole moment associated with $i$ th MO, respectively.

For charged molecules, the first term in the right-hand side $\left(Q_{i} V^{0}\right)$ is dominative. In particular for highly symmetric system, the orbital shift may be simplified only with this $\varepsilon_{i}^{\mathrm{UV}}$ because of the absence of the contribution from polarization. The other two terms $\left(\varepsilon_{i}^{\mathrm{ee}}\right.$ and $\left.\varepsilon_{i}^{\mathrm{wf}}\right)$ in Eq. (8) are not necessary to be considered. Since the occupation number in a MO, corresponding to $Q_{i}$ is a constant, the energy shift is simply written as (approximation 0),

$$
\Delta \varepsilon_{i}^{\text {approx } 0}=V^{0} .
$$

Note that the electrostatic potential $V^{0}$ is mostly determined from the total charge of the solute molecule as discussed below.

If the solute is neutral molecule, the first term in Eq. (9) is vanished and the second term becomes the leading one, in which $\mathbf{R}^{0}$ is the reaction field corresponding to the total dipole moment of the solute molecule in isolated state $\left(\boldsymbol{\mu}^{0}\right)$. To

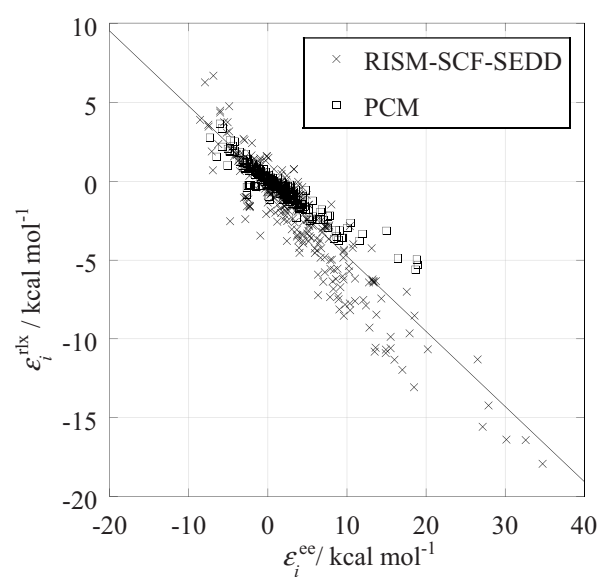

FIG. 4. Comparison between $\varepsilon_{i}^{\text {ee }}$ and $\varepsilon_{i}^{\text {rlx }}$, both of them are computed by PCM and RISM-SCF-SEDD. The slope of the plotted line is -0.476 . See the text and Eq. (14).

study the two terms neglected in Eq. (8) -namely, $\varepsilon_{i}^{\text {rlx }}$ and $\varepsilon_{i}^{\mathrm{ee}}$ - the relationship between them is plotted in Fig. 4. Both of PCM and RISM-SCF-SEDD computational results clearly show a strong correlation between these two quantities. It should be emphasized that the MOs examined here are gathered from a variety of organic compounds. Within the framework of Onsager's dielectric continuum model, this can be explained as follows: the change in reaction field $(\Delta \mathbf{R})$ come from the density change is given by ${ }^{29}$

$$
\Delta \mathbf{R}=-\frac{2(\epsilon-1)}{2 \epsilon+1} \frac{\Delta \mu}{a^{3}},
$$

where $\epsilon$ is the dielectric constant of solvent, $a$ is the cavity radius, and $\Delta \boldsymbol{\mu}$ is the change in dipole moment of the solute molecule. By comparison of Eq. (11) with Eq. (5), $\varepsilon_{i}^{\text {rlx }}$ can be related to the quantity

$$
\varepsilon_{i}^{\mathrm{rlx}} \sim \boldsymbol{\mu}_{i}^{0} \cdot \Delta \mathbf{R}=-\frac{2(\epsilon-1)}{2 \epsilon+1} \frac{\boldsymbol{\mu}_{i}^{0} \cdot \Delta \boldsymbol{\mu}}{a^{3}} .
$$

In a similar manner, $\varepsilon_{i}^{\mathrm{ee}}$ is the change in the Fock operator, which is linked to the change of electronic energy. On the analogy of the expression of the polarization cost using the polarizability of the solute molecule $(\alpha)$,

$$
\varepsilon_{i}^{\mathrm{ee}} \sim \frac{\boldsymbol{\mu}_{i}^{0} \cdot \Delta \boldsymbol{\mu}}{2 \alpha} .
$$

As seen in the definition, the contribution is an expectation value of wave function and not proportional to $(\Delta \mu)^{2}$ because such a contribution is included in $\varepsilon_{i}^{\mathrm{wf}}$. We then reached the following equation using the relationship: ${ }^{30}$

$$
\varepsilon_{i}^{\mathrm{rlx}} \sim-\frac{4(\epsilon-1)}{2 \epsilon+1} \frac{\alpha}{a^{3}} \varepsilon_{i}^{\mathrm{ee}}=-\frac{4(\epsilon-1)}{2 \epsilon+1} \frac{n^{2}-1}{n^{2}+2} \varepsilon_{i}^{\mathrm{ee}} .
$$

Note that the solute-dependent parameters, $\alpha$ and $a$, can be removed from the equation. The proportional constant involves just two parameters, $n$ and $\epsilon$, where $n$ is the refractive index of the solute molecule and typically about 1.4 for almost all organic compounds. ${ }^{31}$ Both of the parameters are then virtually regarded as constant values, independent on the choice of solute molecule. From Eq. (14) with $n=1.4$ and 


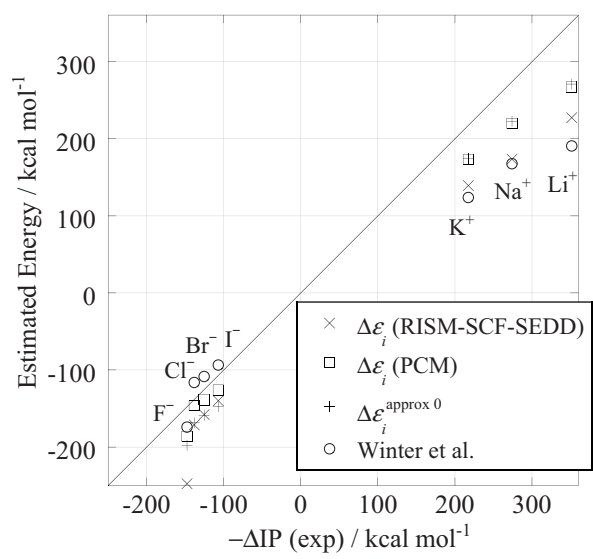

FIG. 5. The shift computed by PCM, RISM-SCF-SEDD as well as the approximation formula of Eq. (20), in comparison with the experimental values, together with the values reported by Winter et al. (Ref. 3).

$\epsilon=80.0$, the slope is determined as -0.476 . As seen in the figure, this value coincides with the distribution for examined 265 MOs. Using this relationship, Eq. (8) can be then rewritten as an approximated form that is valid for many organic compounds in aqueous solution (approximation I),

$$
\begin{aligned}
\Delta \varepsilon_{i}^{\mathrm{approx} \mathrm{I}} & \simeq \varepsilon_{i}^{\mathrm{UV}}+\left\{1-\frac{2 \epsilon+1}{4(\epsilon-1)} \frac{n^{2}+2}{n^{2}-1}\right\} \varepsilon_{i}^{\mathrm{rlx}} \\
& =\varepsilon_{i}^{\mathrm{UV}}+C_{\mathrm{I}} \varepsilon_{i}^{\mathrm{rlx}},
\end{aligned}
$$

where $C_{\mathrm{I}}$ is equal to -1.102 for organic compounds in aqueous solution. Alternatively we can derive (approximation II),

$$
\begin{aligned}
\Delta \varepsilon_{i}^{\mathrm{approx} \mathrm{II}} & \simeq \varepsilon_{i}^{\mathrm{UV}}+\left\{-\frac{4(\epsilon-1)}{2 \epsilon+1} \frac{n^{2}-1}{n^{2}+2}+1\right\} \varepsilon_{i}^{\mathrm{ee}} \\
& =\varepsilon_{i}^{\mathrm{UV}}+C_{\mathrm{II}} \varepsilon_{i}^{\mathrm{ee}} .
\end{aligned}
$$

$C_{\mathrm{II}}$ equals +0.524 for the same conditions.

Since the increase in the dipole moment by solvation is given by Onsager's model,

$$
\Delta \boldsymbol{\mu}=\left\{\frac{\left(n^{2}+2\right)(2 \epsilon+1)}{3\left(2 \epsilon+n^{2}\right)}-1\right\} \boldsymbol{\mu}^{0}=\frac{2}{3} \frac{\left(n^{2}-1\right)(\epsilon-1)}{2 \epsilon+n^{2}} \boldsymbol{\mu}^{0},
$$

and the reaction field is proportional to this quantity, the orbital energy shift might be further simplified only with using $\varepsilon_{i}^{\mathrm{UV}}$ (approximation III),

$$
\begin{aligned}
\Delta \varepsilon_{i}^{\mathrm{approx}} \mathrm{III} \simeq & {\left[1+\left\{1-\frac{2 \epsilon+1}{4(\epsilon-1)} \frac{n^{2}+2}{n^{2}-1}\right\}\right.} \\
& \left.\times \frac{2}{3} \frac{\left(n^{2}-1\right)(\epsilon-1)}{2 \epsilon+n^{2}}\right] \varepsilon_{i}^{\mathrm{UV}} \\
= & \frac{(2 \epsilon+1)\left(n^{2}+2\right)}{6\left(2 \epsilon+n^{2}\right)} \varepsilon_{i}^{\mathrm{UV}}=C_{\mathrm{III}} \varepsilon_{i}^{\mathrm{UV}},
\end{aligned}
$$

where $C_{\mathrm{III}}=0.656$. In this form the shift can be estimated only from the information of gas-phase electronic structure. Remember that Gao et al. ${ }^{13}$ discussed induced dipole moment of molecules in aqueous solution based on $\mathrm{QM} / \mathrm{MM}$ simulations. They found that the dipole moment of organic compound in aqueous solution is about 1.3 times larger than

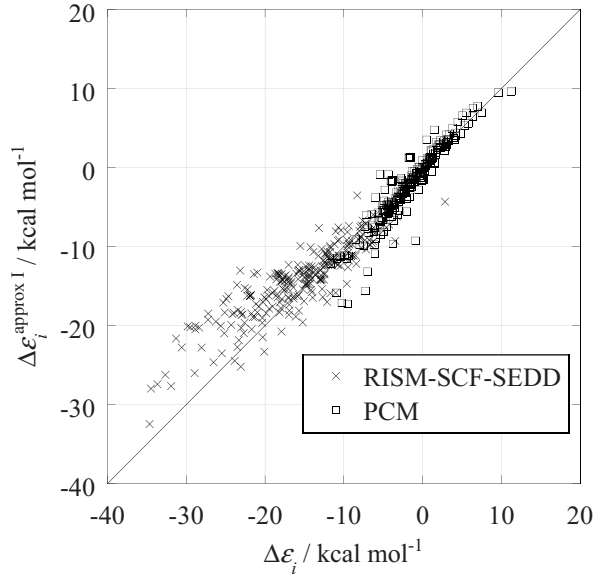

FIG. 6. Comparison between the exact values $\left(\Delta \varepsilon_{i}\right)$ and the approximation formula of Eq. (15).

that in gas phase for a variety of organic compounds. Actually, the prefactor of the right hand side of Eq. (17) is 0.31 by using $n=1.4$ and $\epsilon=80.0$, which shows excellent agreement with their report.

\section{COMPUTATIONAL RESULTS AND DISCUSSIONS}

\section{A. Ionic species in aqueous solution}

Winter $e t a l .^{3}$ recently observed the IP of several ions in aqueous solution. Here we consider ionization of several atomic species in aqueous solution as follows:

$$
\begin{aligned}
& M^{+} \rightarrow M^{2+}+e^{-} \quad(M=\mathrm{Li}, \mathrm{Na}, \mathrm{K}), \\
& A^{-} \rightarrow A+e^{-} \quad(A=\mathrm{F}, \mathrm{Cl}, \mathrm{Br}, \mathrm{I}) .
\end{aligned}
$$

According to Koopmans' theorem, the orbital energies computed by RISM-SCF-SEDD and PCM are regarded as the reversed sign of IPs. The change in IPs by solvation $(\Delta \mathrm{IP})$ is calculated as the difference between the orbital energies computed by both of the methods,

$$
-\Delta \mathrm{IP}=\Delta \varepsilon_{i}^{\mathrm{PCM}} \text { or } \quad-\Delta \mathrm{IP}=\Delta \varepsilon_{i}^{\mathrm{RISM}-\mathrm{SCF}} .
$$

As an example, computed IPs of $\mathrm{Cl}^{-}$in gas phase are $4.1 \mathrm{eV}$ (Koopmans) and $3.2 \mathrm{eV}(\Delta \mathrm{MP} 2),{ }^{32}$ respectively. In aqueous solution, the evaluated values are $11.7 \mathrm{eV}$ (Koopmans) and $10.9 \mathrm{eV}(\Delta \mathrm{MP} 2)$, indicating the difference between the two methods is similar in magnitude to the gas phase. This trend is also found in polyatomic molecular systems. The most important is the difference is much smaller than the effect from solvation, especially for ionic species, and the discussion based on Koopmans' theorem is expected to provide correct understanding in semiquantitative sense.

In the present simplest system, an equivalent quantity can be evaluated from the above mentioned "approximation 0." By using Born approximation with ionic radius $a$, the shift is written as

$$
\Delta \varepsilon_{i}^{\text {approx } 0}=V^{0} \simeq\left(1-\frac{1}{\epsilon}\right) \frac{Q}{a},
$$

where $Q$ is total charge of the ionic species. $a$ 's are taken from the PCM parameters. Figure 5 compares Eq. (20) with 


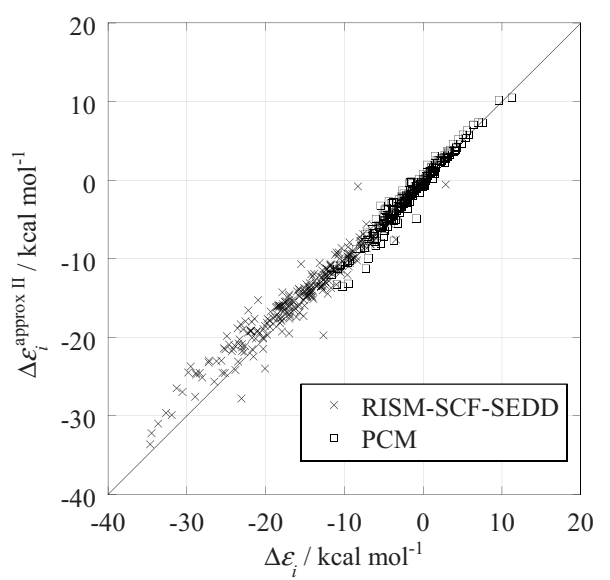

FIG. 7. Comparison between the exact values $\left(\Delta \varepsilon_{i}\right)$ and the approximation formula of Eq. (16).

corresponding experimental values. A good agreement is seen in all data; $a b$ initio RISM-SCF-SEDD and PCM can reproduce the experimental data while the simplest formula also shows good accordance with experiments and highly sophisticated theoretical methods. Some of the plots evaluated from Eq. (20) coincide with PCM values very well because both of them are based on the dielectric continuum theory. The present estimations also show an excellent agreement with the values reported by Winter $e t a l^{3}$

\section{B. Verification of the approximations}

We would like to check accuracy of the introduced approximations for other ordinary polyatomic molecules. Similar to the treatment on Eq. (8), the exact value of the shift $\left(\Delta \varepsilon_{i}\right)$ is compared to the formulas, $\Delta \varepsilon_{i}^{\text {approx I }}$ (Fig. 6),

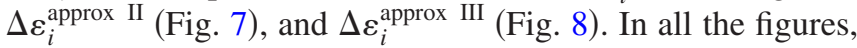
results from PCM and RISM-SCF-SEDD are plotted all together. In any case, the approximated formulas work well and the shift can be understood over a variety of MOs. The approximation II looks slightly better than approximation I presumably because $\varepsilon_{i}^{\mathrm{ee}}$ used in this expression is a direct quantity related to the electronic structure of solute molecule while $\varepsilon_{i}^{\text {rlx }}$ is the electrostatic interaction from solvent field, which indirectly associated with the orbital property.

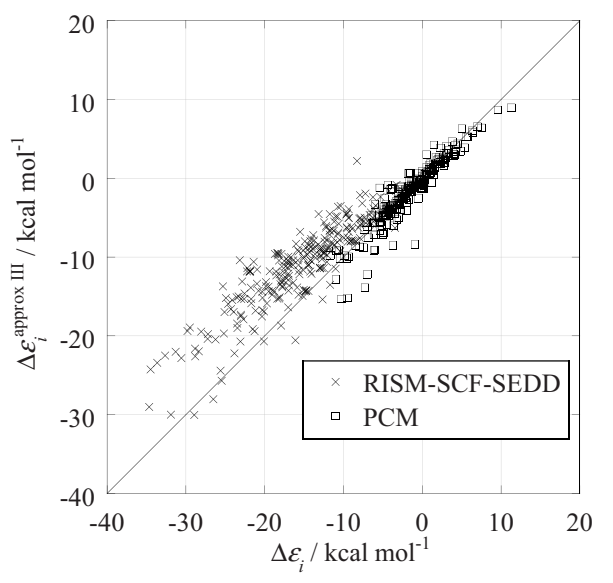

FIG. 8. Comparison between the exact values $\left(\Delta \varepsilon_{i}\right)$ and the approximation formula of Eq. (18).
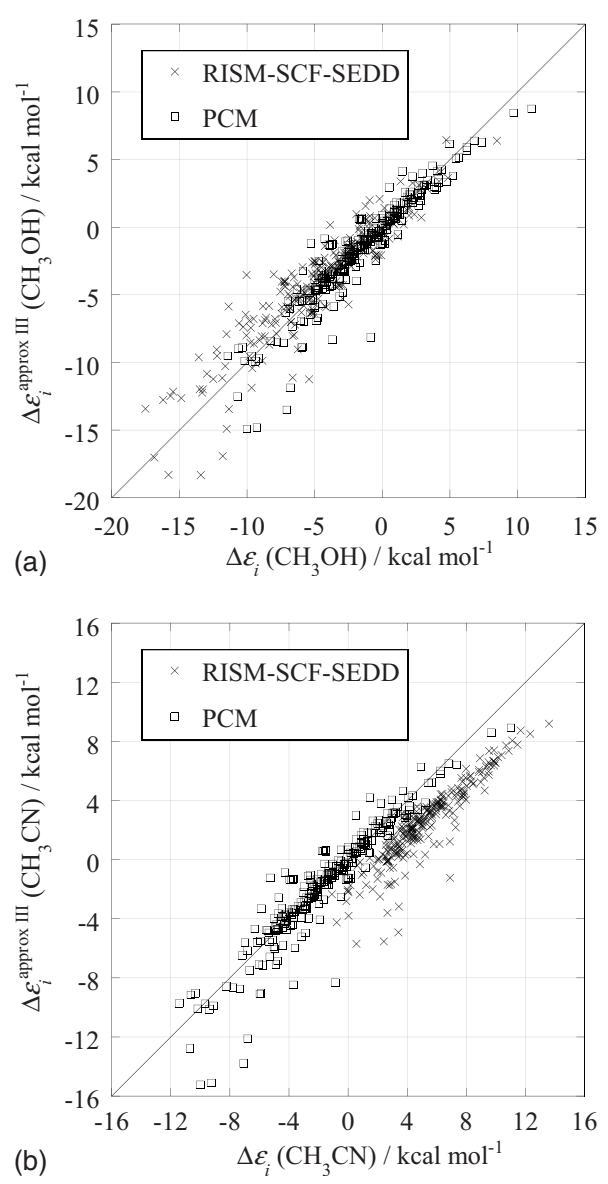

FIG. 9. (a) The energy shift in methanol. The exact values $\left(\Delta \varepsilon_{i}\right)$ and the approximation formula of Eq. (18) are compared. (b) The energy shift in acetonitrile. The exact values $\left(\Delta \varepsilon_{i}\right)$ and the approximation formula of Eq. (18) are compared.

Approximation III is the lowest level of approximation that uses just single-species matrix elements, $\varepsilon_{i}^{\mathrm{UV}}$. Even with this simple expression, the estimated shifts are satisfactory, strongly indicating that the orbital energy shift is essentially derived from the electrostatic interaction acting on MO. Good agreement between the two solvation methods indicates that the derived approximations are universal and applicable to other type of solvation theories.

\section{Orbital energy shift in various solvents}

The obtained relationships are also valid for other solvents. Figure 9 shows the exact shifts in (a) methanol and in (b) acetonitrile compared to the approximation III formula. The dielectric constants are 32.62 (methanol) and 36.64 (acetonitrile), leading to 0.651 and 0.652 of $C_{\mathrm{III}}$, respectively. Again, good agreements are found in all the cases: the orbital energy shifts in methanol computed from the two methods are plotted in narrow confine. The result in acetonitrile solvent is also similar, but somewhat interesting. The values computed by RISM-SCF-SEDD tend to be distributed at the right-hand side of the line, suggesting that the electrostatic solute-solvent interactions evaluated by RISM-SCF-SEDD are slightly stronger than those by the dielectric continuum theory. We would like to remind the readers that it makes sense that the PCM results are closer to the line because the 
approximated formula is derived with the aid of dielectric continuum model. It would be possible that the RISM-SCFSEDD results are closer to the "experimental" valueswhich are not available at this moment-because RISMSCF-SEDD is based on the atomic level interaction between molecules, and specific coordination and local structure such as hydrogen bonding are explicitly treated. There is still controversy over the liquid structure of acetonitrile but it is very plausible that strong electric field is generated in this highly polar solvent.

The main difference between PCM and RISM-SCFSEDD is usually attributed to the capability to describe such specific solvation structure. As seen in Eq. (18), the shift is predictable if the electrostatic interaction is known,

$$
C_{\mathrm{III}} \varepsilon_{i}^{\mathrm{UV}}=C_{\mathrm{III}}\left\langle\psi_{i}^{0}\left|\hat{V}^{0}\right| \psi_{i}^{0}\right\rangle
$$

Note that all the information in the last term contains only gas-phase quantities, meaning that the shift can be estimated, in principle, only from the electronic structure obtained by standard MO method. However, in practice, determination of $\hat{V}^{0}$ is not simple and depends on the solvation methods. ${ }^{33,34}$ In the RISM-SCF-SEDD theory, the potential operator of solvation effect is evaluated from PCFs between the solute site $A$ and solvent $s, g_{A s}(r)$.

$$
\hat{V}_{(\mathrm{RISM}-\mathrm{SCF})}=\sum_{A} \hat{b}_{A} \sum_{s} \rho \int_{0}^{\infty} 4 \pi r^{2} g_{A s}(r) \frac{q_{s}}{r} d r,
$$

where $\hat{b}_{A}$ is an appropriate population operator on atom $A .^{8}$ In evaluation of $\hat{V}^{0}$, PCFs were evaluated from the electronic structure fixed to isolated molecule $\left(g_{A s}^{0}(r)\right)$. The corresponding operator in PCM theory is evaluated from the effective charges of each tesserae on the boundary surface, $\sigma_{t}$,

$$
\hat{V}_{(\mathrm{PCM})}=\sum_{t}^{\text {tesserae }} \frac{\sigma_{t}}{\left|\mathbf{r}-\mathbf{r}_{t}\right|}
$$

In a similar manner, effective charge corresponding to the electronic structure in gas phase is $\sigma_{t}^{0}$. All the quantities could be computed if $\left\{\psi_{i}^{0}\right\}$ are available, but actual treatment of $\hat{V}^{0}$ differs in both methods. It is interesting that complicated modern computational results can be explained by unified formulas based on the simplest Onsager's theory. The dipole moment based expression can reasonably represent the solute-solvent interaction at least in the present set of molecules.

\section{Solvation structure and energy shift}

Finally, we would like to get back to the orbital energy shifts of acetamide shown in Sec. I. As shown in Fig. 1, orbital numbers 1 (oxygen core), 5 ( $\sigma$-bond), and 15 (lone pair) are greatly affected by solvation. Based on Eqs. (9) and (18), the shift must be attributed to the interaction between the $i$ th orbital dipole moment $\left(\boldsymbol{\mu}_{i}\right)$ (Ref. 35) and total dipole moment of the molecule $\left(\boldsymbol{\mu}^{0}\right)$,

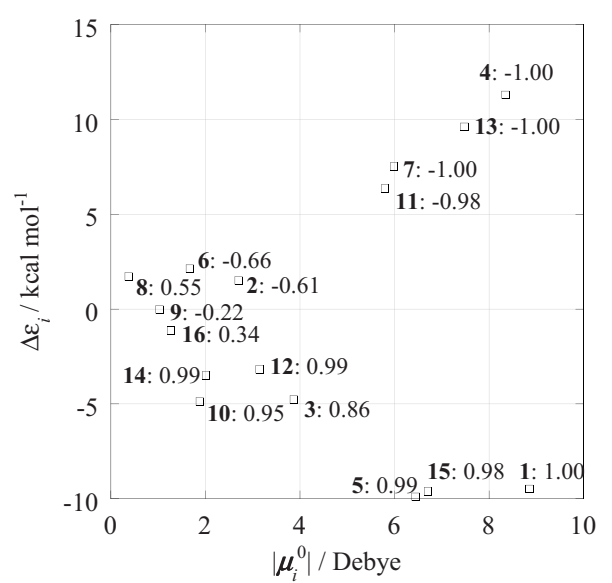

FIG. 10. Orbital energy shift in acetamide compared with the absolute value of dipole moment attributed to each $\mathrm{MO}\left(\boldsymbol{\mu}_{i}^{0}\right)$. The orbital number and $\cos \theta_{i}$ are also shown.

$$
\begin{aligned}
\varepsilon_{i}^{\mathrm{UV}} \simeq \boldsymbol{\mu}_{i}^{0} \cdot \mathbf{R}^{0}= & -\frac{2(\epsilon-1)}{2 \epsilon+1} \frac{\boldsymbol{\mu}_{i}^{0} \cdot \boldsymbol{\mu}^{0}}{a^{3}} \\
& =-\frac{2(\epsilon-1)}{2 \epsilon+1} \frac{\left|\boldsymbol{\mu}_{i}^{0} \| \boldsymbol{\mu}^{0}\right| \cos \theta_{i}}{a^{3}}
\end{aligned}
$$

where $\theta_{i}$ is the angle between $\boldsymbol{\mu}_{i}$ and the total dipole moment. $\boldsymbol{\mu}_{i}$ for the three orbitals are 8.9, 6.4, and 6.7 D, respectively. As seen in Fig. 1, larger amplitudes in the 1st, 5th, and 15 th orbitals are found near oxygen atom, which is one of the keys to make the orbital shifted.

Another important aspects drawn from the equation is the relative directions of the two dipole moments (see Fig. $10)$. For example, $\left|\boldsymbol{\mu}_{4}\right|$ is large enough $(8.4 \mathrm{D})$ but its direction is almost opposite to that of the total dipole moment of the molecule, $\boldsymbol{\mu}^{0}$. As a result, the orbital energy is positively shifted. In summary, combination of spatial extension of the MO and electrostatic field generated by surrounding solvent governs the energy shift of MOs.

\section{CONCLUSIONS}

We present a systematic understanding for energy shift of MO by solvation effect. With the aid of dielectric continuum theory, several simple formulae are developed to rationalize the orbital energy shifts. For charged system, Borntype treatment is acceptable to estimate the orbital shift. At the same time, approximated formulae based on the Onsagertype model are satisfactory for examined 265 MOs of neutral species. We found that both of the spatial distribution of MO and electrostatic field generated by surrounding solvent are important to determine the orbital energy shift. The developed formulae are valid to explain both for PCM and RISMSCF-SEDD computations, strongly indicating the discussion in this study is a common consequence to understand the electronic structure of solvated molecule, being independent of the choice of solvation theories.

The orbital shift is not a direct observable but deeply related to IP and/or electron affinity via Koopmans' theorem. In the forthcoming paper, the relationship between the experimental measurements and computational evaluation for solvated molecule will be extensively discussed. 


\section{ACKNOWLEDGMENTS}

This work was financially supported by the Grant-in Aids (Nos. 461, 19350010, 20550013, and 452-20031014), all supported by the Ministry of Education, Culture, Sports, Science and Technology (MEXT) Japan.

${ }^{1}$ A. Szabo and N. S. Ostlund, Modern Quantum Chemistry (MacMillan, New York, 1982).

${ }^{2}$ B. Winter, R. Weber, W. Widdra, M. Dittmar, M. Faubel, and I. V. Hertel, J. Chem. Phys. 108, 2625 (2004).

${ }^{3}$ B. Winter, R. Weber, I. V. Hertel, M. Faubel, P. Jungwirth, E. C. Brown, and S. E. Bradforth, J. Am. Chem. Soc. 127, 7203 (2005).

${ }^{4}$ K. Iida, D. Yokogawa, H. Sato, and S. Sakaki, Chem. Phys. Lett. 443, 264 (2007).

${ }^{5}$ R. R. Contreras and A. J. Aizman, Int. J. Quantum Chem. 24, 89 (1990).

${ }^{6}$ G. Alagona, C. Ghio, J. Igual, and J. Tomasi, J. Mol. Struct.: THEOCHEM 204, 253 (1990).

${ }^{7}$ E. S. Marcos, J. Maraver, M. F. Ruiz-Lopez, and J. Bertran, Can. J. Chem. 64, 2353 (1986)

${ }^{8}$ S. Ten-no, F. Hirata, and S. Kato, J. Chem. Phys. 100, 7443 (1994).

${ }^{9}$ H. Sato, F. Hirata, and S. Kato, J. Chem. Phys. 105, 1546 (1996).

${ }^{10}$ D. Yokogawa, H. Sato, and S. Sakaki, J. Chem. Phys. 126, 244504 (2007).

${ }^{11}$ J. Tomasi and M. Persico, Chem. Rev. (Washington, D.C.) 94, 2027 (1994).

${ }^{12}$ J. Gao and X. Xia, Science 258, 631 (1992).

${ }^{13}$ J. Gao, F. J. Luque, and M. Orozo, J. Chem. Phys. 98, 2975 (1993).

${ }^{14}$ For Br, see L. A. Curtiss, M. P. McGrath, J.-P. Blaudeau, N. E. Davis, R. C. Binning, Jr., and L. Radom, J. Chem. Phys. 103, 6104 (1995); for I, see M. N. Glukhovtsev, A. Pross, M. P. McGrath, and L. Radom, ibid. 103, 1878 (1995)

${ }^{15}$ D. Elking, T. Darden, and R. J. Woods, J. Comput. Chem. 28, 1261 (2007).

${ }^{16}$ S. J. Singer and D. Chandler, Mol. Phys. 55, 621 (1985).

${ }^{17}$ S. J. Weiner, P. A. Kollman, D. A. Case, U. C. Singh, C. Ghio,
G. Alagona, S. Profeta, Jr., and P. Weiner, J. Am. Chem. Soc. 106, 765 (1984).

${ }^{18}$ S. J. Weiner, P. A. Kollman, D. T. Nguyen, and D. A. Case, J. Comput. Chem. 7, 230 (1986).

${ }^{19}$ R. C. Rizzo and W. L. Jorgensen, J. Am. Chem. Soc. 121, 4827 (1999).

${ }^{20}$ M. Kinoshita and F. Hirata, J. Chem. Phys. 106, 5202 (1997).

${ }^{21}$ B. M. Pettitt and P. J. Rossky, J. Chem. Phys. 84, 5836 (1986).

${ }^{22}$ H. J. C. Berendsen, J. P. M. Postma, W. F. van Gunsteren, and J. Hermans, in Intermolecular Forces, edited by B. Pullman (Reidel, Dordrecht, 1981).

${ }^{23}$ W. L. Jorgensen and J. M. Briggs, Mol. Phys. 63, 547 (1988).

${ }^{24}$ W. L. Jorgensen, J. Phys. Chem. 90, 1276 (1986)

${ }^{25}$ W. L. Jorgensen, J. D. Madura, and C. J. Swenson, J. Am. Chem. Soc. 106, 6638 (1984)

${ }^{26}$ M. W. Schmidt, K. K. Baldridge, J. A. Boatz, S. T. Elbert, M. S. Gordon, J. H. Jensen, S. Koseki, N. Matsunaga, K. A. Nguyen, S. Su, T. L. Windus, M. Dupuis, and J. A. Montgomery, J. Comput. Chem. 14, 1347 (1993).

${ }^{27}$ M. J. Frisch, G. W. Trucks, H. B. Schlegel et al., Gaussian 03, Revision C.02, Gaussian, Inc., Wallingford, CT, 2004.

${ }^{28}$ F. J. Luque, J. M. Bofill, and M. Orozco, J. Chem. Phys. 103, 10183 (1995).

${ }^{29}$ C. J. F. Bottcher, Theory of Electric Polarization (Elsevier, Amsterdam, 1983).

${ }^{30}$ J. N. Wilson, Chem. Rev. (Washington, D.C.) 25, 377 (1939).

${ }^{31}$ K. Sharp, A. Jean-Charles, and B. Honig, J. Phys. Chem. 96, 3822 (1992).

${ }^{32}$ The energy difference between the two states were computed by MP2 and UMP2 method. When applying $\triangle$ SCF methods, computed values were $2.5 \mathrm{eV}$ (gas) and $10.2 \mathrm{eV}$ (aqueous). The good agreement with $\triangle \mathrm{MP} 2$ comes from the well-known cancellation of electron correlation and the orbital relaxation in Koopmans' theorem (Ref. 1) which does not occur in $\triangle \mathrm{SCF}$.

${ }^{33}$ Continuum Solvation Models in Chemical Physics: From Theory to Applications, edited by B. Mennucci and R. Cammi (Wiley, New York, 2007).

${ }^{34}$ H. Sato and S. Sakaki, J. Phys. Chem. A 108, 1629 (2004).

${ }^{35}$ The origin was set to the center of nuclear charges. 